\title{
Computerised Retinal Image Analysis to Detect and Quantify Exudates Associated with Diabetic Retinopathy
}

\author{
M. Ponni Bala \\ Assistant Professor(SRG) \\ Kongu Engineering College \\ Perundurai, Erode, Tamilnadu
}

\author{
S. Vijayachitra, Ph.D \\ Professor \\ Kongu Engineering College \\ Perundurai, Erode, Tamilnadu
}

\begin{abstract}
One of the greatest concern and immediate challenges to the current health care is the severe progression of diabetes. Diabetic retinopathy is an eye disease that associated with long-standing diabetes. The conventional method followed by ophthalmogists is the regular supervision of the retina. As this method takes time and energy of the ophthalmogists, a new feature based automated technique for classification and detection of exudates in color fundus image is proposed in this paper. This method reduces the professionals work to examine on every fundus image rather than only on abnormal image. The exudates are separated from the fundus image by thresholding and removal of optic disk using morphological operation and connected component analysis. Finally, an automated Fuzzy Inference System (FIS) is used for classifying the retinal images as exudates and its severity and non-exudates. The sensitivity, specificity and accuracy are reported as $91.11 \%, 100 \%$ and $93.84 \%$ for Fuzzy Inference System Classification.
\end{abstract}

\section{General Terms}

Image Classification, Fuzzy Inference System

\section{Keywords}

Exudates, Fundus image, connected component, Morphological operation, Fuzzy Inference System.

\section{INTRODUCTION}

Diabetic Retinopathy (DR) is the eye related disease caused by the diabetes. DR is the most common sight threatening disease when untreated leads to vision loss and in many cases it cannot be reversed. There are two types of DR: NonProliferative diabetic retinopathy (NPDR) and Proliferative diabetic retinopathy (PDR). In NPDR, the damaged blood vessels leak extra fluid and small amount of blood into the eye. This condition leads to the formation of exudates in the retina. As the disease progresses the amount of exudates also increases. In PDR, the blood vessels in the retina close and prevent blood flow in the eye. This condition leads to the formation of new blood vessels in order to supply blood to the blocked area and this condition is called as neovascularization.

The normal retinal image and the retinal image with exudates are shown in Fig. 1 (a) and (b). Exudates are manifested as spatially random yellowish or whitish patches of varying sizes, shapes and locations. These are the visible sign of DR and a major cause of visual loss in Non-Proliferative forms of DR. In this work, the retinal images are classified as exudates and non-exudates using disease based features and statistical features extracted from the images.

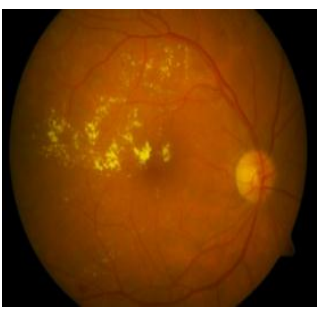

(a)

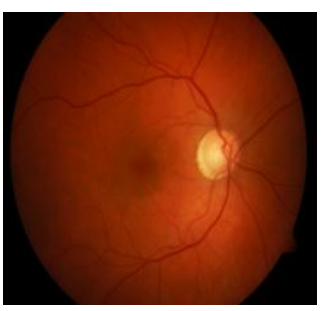

(b)
Fig1: a) Normal Retinal Image b) Retinal image with exudates

\section{LITERATURE REVIEW}

The detection of exudates in retinal images was investigated by many researchers as follows. Alireza Osareh et al. [1] developed a tool to segment the retinal images using Fuzzy CMeans (FCM) clustering technique. The features are extracted and ranked using Genetic Algorithm and classified using neural network. A k a a Sop hara k et al. [2] detected exudates from nonmydriatic, low contrast retinal digital images using mathematical morphology techniques. Akara Sopharak et al.[3] proposed an automatic method for detection of exudates from the diabetic retinopathy images using fuzzy c-means clustering technique. Asha Gowda Karegowda et al. [4] developed a Back propagation Neural network classifier to detect exudates in retinal images. The significant features are identified by Decision Tree and GACFS method. Doaa Youssef et al. [5] found that Green channel have high contrast and necessary information (exudates). Canny edge detector and Hough transform was used for optic disk elimination and morphological operation for detection of blood vessel tree. A Bayesian classifier was used for classification of images into exudates and nonexudates was given by Ege et al. [6].

Huan Wang et al. [7] detected exudates by using a minimumdistance discriminant classifier based on statistical pattern recognition and used local window for classification. Lili $\mathrm{Xu}$ et al. [9] use a segmentation method to differentiate the contrast in larger and thin blood vessels. Adaptive local thresholding is used to produce the normalized image and to extract larger vessels. Thin vessel segments are classified using Support Vector Machine. Different stages of Diabetic retinopathy disease severity are detected by Morphological operation and Texture Analysis methods applied on retinal images was found by [11], [13]. The statistical features are extracted and classified using Bayes Minimum Distant Discriminant (MDD) classifier and the classifier is compared with original and brightness enhanced image is shown in [10], [14]. Niemeijer et al. [12] proposed a method to differentiate the bright lesions such as exudates, cotton wool spots and drusen from colour retinal images. Sinthanayothin C et al. [16] developed a Recursive region growing segmentation 
technique which is used for the automated system of detection of diabetic retinopathy stages. Huiqi Li and Opas Chutatape [8] have presented a method to detect exudates using region growing and edge detection techniques. He also detected optic disk using principal component analysis. Using a modified active shape model the shape of optic disk was detected. Sánchez et al. [15] detected hard exudates by colour, statistical classification and sharpness of its edges using Kirsch operator.

A fundus coordinate system is used to describe the features. Usher et al. [17] found an adaptive intensity thresholding method for the extraction of exudates. The features extracted are size, shape, hue and intensity and are used as the input to artificial neural network. Prof B. Venkatalakshmi and V.Saravanan [18] presented a method to detect hard exudates using color and sharp edges of lesion. Graphical user interface window created using MATLAB used in examining the abnormal fundal retinal images automatically and thus reducing the examining time of the physician. Walter et al. [19] has presented and discussed an algorithm for detection of exudates, as well as detection of optic disk which was essential for this approach. Exudates were found using their grey level variation, and their contours were determined by means of morphological reconstruction techniques. The optic disk was detected by means of morphological filtering techniques and the watershed transformation was to find its contours. V.Vijaya Kumari, N.SuriyaNarayanan [20] developed a method for early detection of Diabetic Retinopathy. Optic disk is extracted by propagation through radii method and exudates are detected using feature extraction, template matching and enhanced MDD classifier.

\section{MATERIALS AND METHODS}

\subsection{Methodology}

The objective of this project work is to classify the retinal image into exudates and its severity stages and non-exudates. The retinal image used for this project work is subjected to the preprocessing steps such as green channel extraction, histogram equalization and contrast enhancement. The optic disk is eliminated by connected component analysis and features like exudates area, size, color, and homogeneity and texture properties are extracted. The block diagram of the proposed method is shown in Fig. 2.

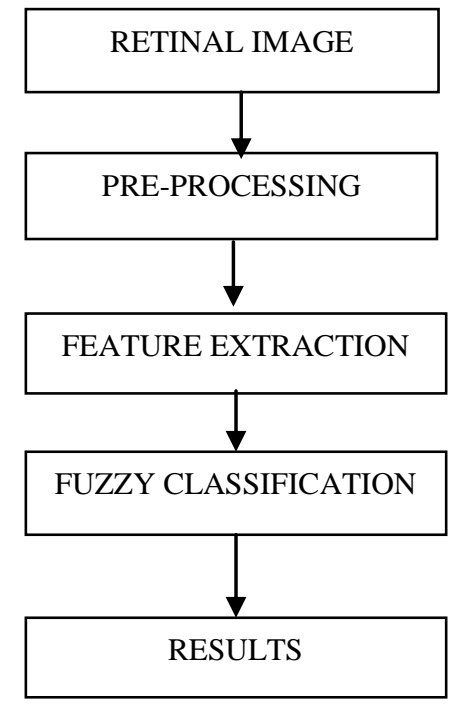

Fig. 2: Block diagram of the proposed method

\subsection{Image Acquisition}

The images for this project work are taken from LOTUS EYE CARE, Coimbatore. The images forms a dataset of 65 colour fundus images in which 20 are normal and 45 contains exudates patches with different stages of severity. Images were captured with 90 degree field-of-view digital fundus Cannon non-mydriatic ZEISS camera. Each image was captured using 24 bit per pixel at a resolution of 774 X893 pixels in JPEG format.

\subsection{Preprocessing}

The retinal images in the dataset are often noisy and poorly illuminated because of unknown noise and camera settings. Also there is a wide variation of colour of retina from patient to patient. Thus the images are subjected to various preprocessing steps, which include green channel extraction, histogram equalization and contrast enhancement. The retinal images and the preprocessed images for normal, mild, moderate and severe stages of exudates are shown in Fig.3 and Fig. 4 respectively.

The exudates appear bright in the green channel compared to red and blue channels in RGB image. Hence green channel is used for further processing by neglecting other two components. Histogram equalization and contrast enhancement are used to increase the contrast between the exudates and the image background.

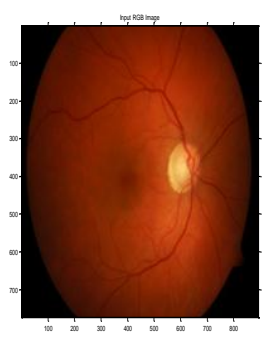

a

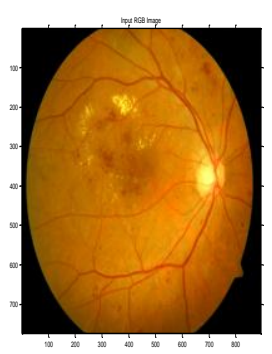

c

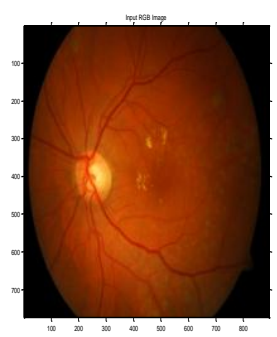

b

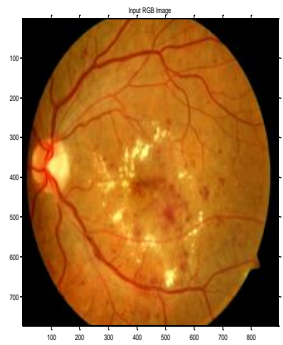

d
Fig.3: Retinal Images with Different Stages of Exudates Severity

a) Normal b) Mild c) Moderate d) Severe 


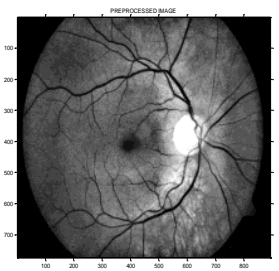

a

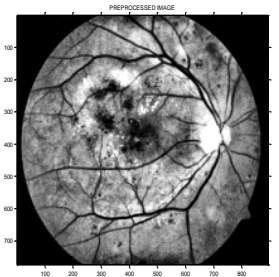

c

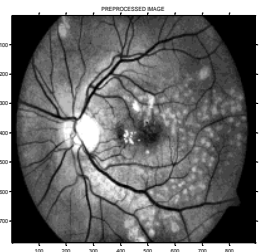

b

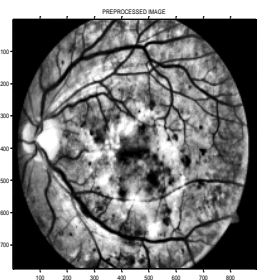

d
Fig.4: Preprocessed Retinal Images

a) Normal b) Mild c) Moderate d) Severe

\subsection{Morphological operation}

The enhanced retinal image is converted to binary image by applying proper thresholding value. This binary image is subjected to morphological operations i.e. opening and closing. Closing operation is defined as dilation and opening as erosion. Dilation is an operation that grows or thickens objects in a binary image. Erosion shrinks or thins the objects in the binary image. The process of thickening and thinning is controlled by a shape called structuring element. As the optic disk and exudates are circular in shape, a disk shape structuring element is used in this project.

\subsection{Optic disk elimination}

The morphologically operated image has only exudates and optic disk. The optic disk occupied maximum area in the image and hence it is eliminated by connected component analysis. The optic disk eliminated image for normal, mild, moderate and severe stages of Diabetic Retinopathy are shown in Fig. 5.

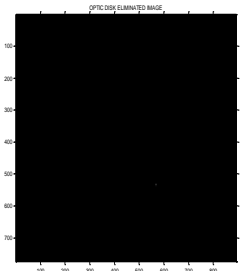

a

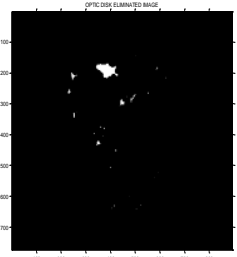

c

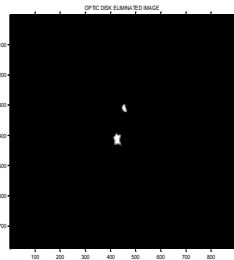

b

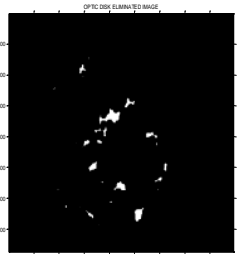

d
Fig.5: Optic Disk Eliminated Images

\subsection{Columnwise operation}

In this operation, the image is separated into blocks and each block is converted into a column in a temporary matrix, and then applies the function (mean, variance, etc) to this matrix. The function return a row vector containing a single value for each column in the temporary matrix. This temporary matrix is again converted into a matrix as same size as the image. This operation gives proper visibility of exudates and main blood vessels in the retinal image. The columnwise operated image for normal, mild, moderate and severe stages of exudates are shown Fig.7.

\section{FEATURE EXTRACTION}

The processed image after the removal of optic disk has only exudates. This image is used for feature extraction. The disease based features such as exudates area, exudates perimeter and number of exudates patches and the statistical features such as mean, standard deviation, energy, contrast, correlation, homogeneity, entropy, cluster shade, cluster prominence, skewness and kurtosis are extracted. From these features, the most effective features are used for classification.
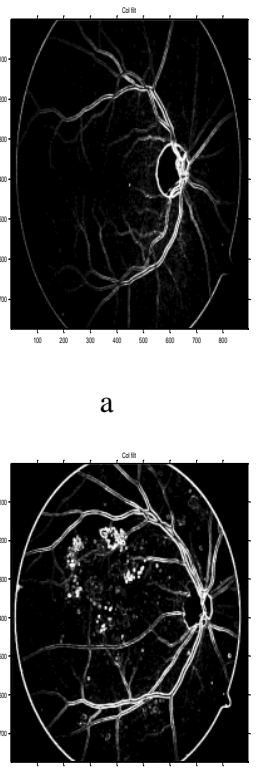

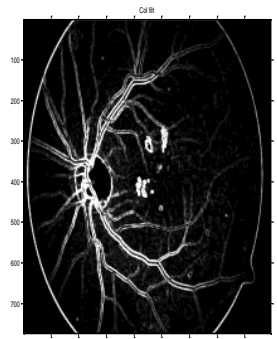

b

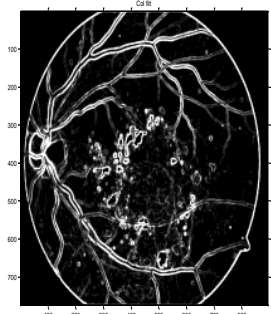

d
Fig.6: Column-wise Operated Images

\section{RESULTS AND DISCUSSION}

The images for this project work are taken from LOTUS EYE CARE, Coimbatore. The retinal image database contains images of various diabetic retinopathy signs (red small dots, haemorrhages, hard exudates, soft exudates and neovascularisation). The retinal image from the dataset is processed and features are extracted.

A set of 65 images are analyzed and the features such as exudates area, exudates perimeter, number of exudates patches, mean, standard deviation, energy, contrast, correlation, homogeneity, entropy, cluster shade, cluster prominence, skewness and kurtosis are extracted. Table 1 shows the feature values extracted from the sample 10 images. 
From the extracted fourteen features, four features are used for classification. A mamdani based Fuzzy Inference System is used as a classifier. It is used for classifying retinal images as four stages namely normal, mild, moderate and severe case.

In addition to classification of images based on exudates and its severity and nonexudates, a MATLAB Graphical User Interface (GUI) based system is developed to quantify the features and display the severity stages. The GUI output for a normal and an abnormal image are shown in Fig.8 and Fig.9 respectively. Thus the system is used to assist Doctors in hospitals.

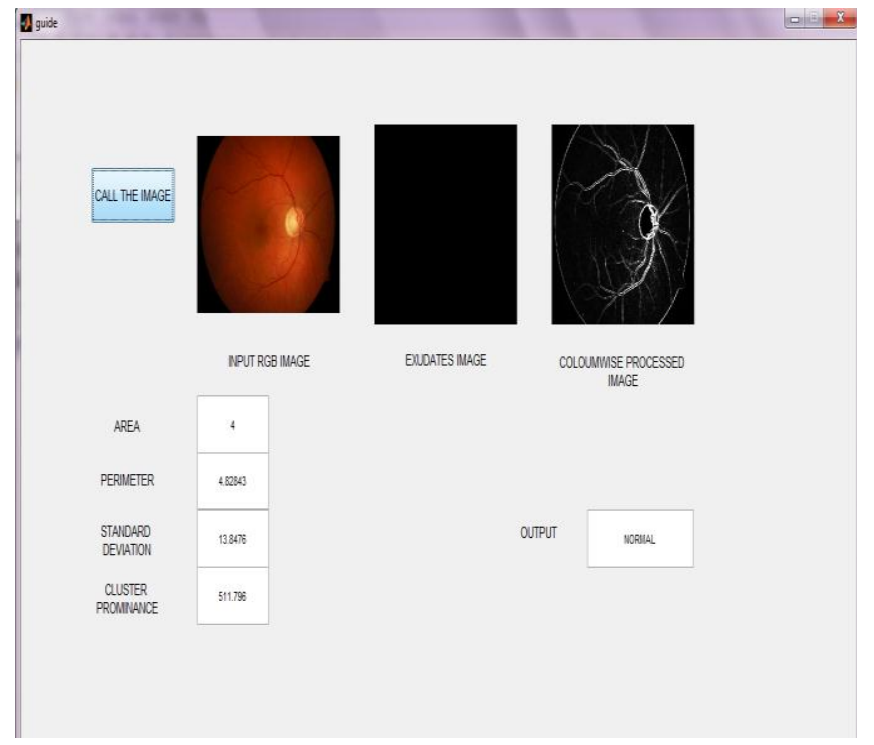

Fig.7: GUI for a normal image

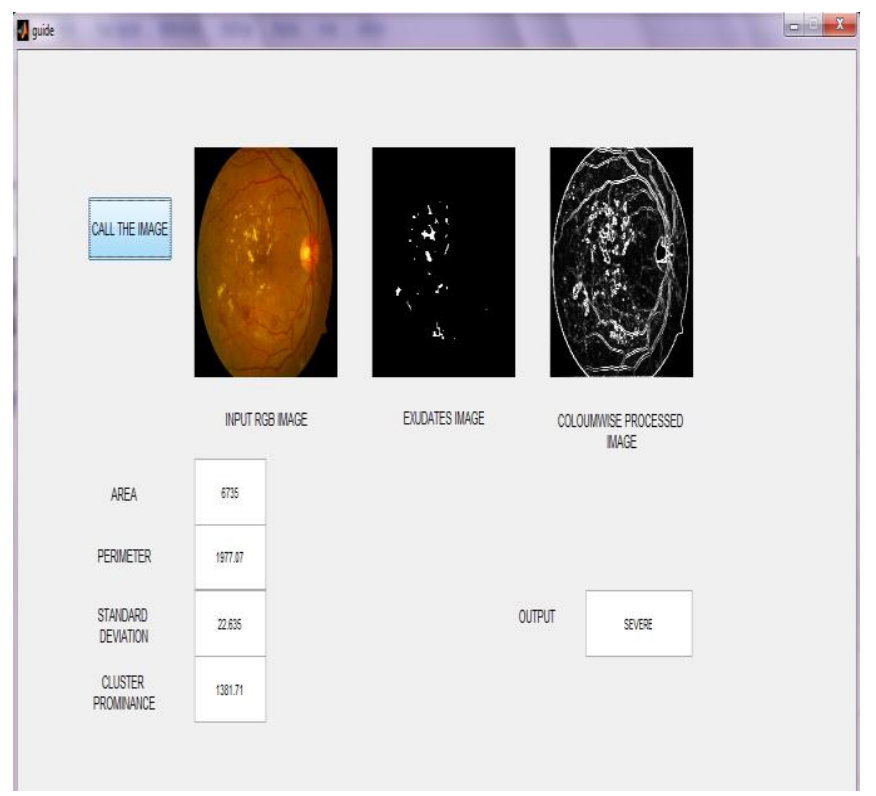

Fig.8: GUI for an abnormal image
Performance of the proposed method is evaluated by calculating the sensitivity, specificity and accuracy percentages by comparing the detection result with ophthalmologist's hand drawn ground truth. The sensitivity, specificity and accuracy are given by,

$$
\begin{aligned}
& \text { Sensitivity }=\frac{T P}{T P+F N} \\
& \text { Specificity }=\frac{T N}{T N+F P} \\
& \text { Accuracy }=\frac{(T P+T N)}{(T P+F P=F N+T N)}
\end{aligned}
$$

This method gives sensitivity, specificity and accuracy of 91.11\%, $100 \%$ and $93.84 \%$ for Fuzzy Inference System Classification.

\section{CONCLUSION}

The main idea of this project is to automatically classify retinal images as exudates and its severity stages and nonexudates image using Fuzzy techniques and GUI from the extracted features. Thus the retinal image is subjected to various processing and features are extracted for a set of 65 images of which 20 are normal and 45 are abnormal images with different stages of severity. The processing steps include green channel extraction, histogram equalization, contrast enhancement, morphological operations, optic disk elimination and column-wise operation. Totally fourteen features are extracted from which four features are taken for classification. Fuzzy Inference System is used for classification. Further, Fuzzy Inference System is automated using Graphical User Interface. The automation helps in reducing the doctor's time by observing only the diseased image rather than all retinal images. The sensitivity specificity and accuracy obtained for FIS are $91.11 \%, 100 \%$ and 93.84 $\%$ respectively, which can be improved further by other soft computing methods in future.

\section{ACKNOWLEDGMENT}

The authors are grateful to Lotus Eye Care Hospital, Coimbatore for providing the photographic database of eye images and also to Dr. R. J. Madhusudanan DO, for his valuable suggestions on this work. 
Table 1 : Results of extracted feature values of Normal and abnormal images

\begin{tabular}{|c|c|c|c|c|}
\hline Images & Exudates Area & Exudates Perimeter & Standard Deviation & $\begin{array}{c}\text { Cluster } \\
\text { Prominence }\end{array}$ \\
\hline 1Normal & 0 & 0 & 12.6636 & 37.1786 \\
\hline 2 Normal & 0 & 0 & 12.4484 & 39.2548 \\
\hline 3 Normal & 0 & 0 & 14.7885 & 56.4474 \\
\hline 4 Normal & 4 & 4.8484 & 13.8476 & 43.9339 \\
\hline 5 Mild & 79 & 63.0122 & 16.8181 & 67.8963 \\
\hline 6 Mild & 1567 & 574.6589 & 17.9293 & 69.6419 \\
\hline 7 Medium & 1640 & 683.5706 & 19.3183 & 94.3429 \\
\hline 8 Medium & 3286 & 1238.2 & 19.9039 & 96.8846 \\
\hline 9 Severe & 5243 & 1668.5 & 22.6175 & 118.5877 \\
\hline 10 Severe & 7836 & 1597 & 20.5315 & 117.1396 \\
\hline
\end{tabular}

\section{REFERENCES}

[1] Alireza Osareh, Bita Shadgar and Richard Markham, 2009 A computational-intelligence-based approach for detection of exudates in diabetic retinopathy images. IEEE Transactions on Information Technology in Biomedicine, Vol. 13, no. 4, pp. 535-545.

[2] Akara Sopharak, Bunyarit Uyyanonvara, Sarah Barman, Thomas H. Williamson, 2008. Automatic detection of diabetic retinopathy exudates from non-dilated retinal images using mathematical morphology methods, Computerized Medical Imaging and Graphics 32, 720727.

[3] Akara Sopharak, Bunyarit Uyyanonvara, Sarah Barman, 2009, "Automatic Exudate Detection for Diabetic Retinopathy Screening”, ScienceAsia, 35, 80-85.

[4] Asha Gowda Karegowda, Asfiya Nasiha, M.A.Jayaram, 2011. Exudates Detection in Retinal Images using Back Propagation Neural Network, International Journal of Computer Applications (0975 - 8887), Volume 25, No.3.

[5] Doaa Youssef, N. Solouma, A. El-dib, M. Mabrouk, and A. B. Youssef , 2010. New Feature-Based Detection of Blood Vessels and Exudates in Color Fundus Images. IEEE International Conf. on Image Processing Theory Tools \& Applications. 294-299.

[6] Ege B.M., Hejlesen O.K., Larsen O.V., Moller K., Jennings B., Kerr D. and Cavan D.A., 2000. Screening for diabetic retinopathy using computer based image analysis and statistical classification. Computer Methods and Programs in Biomedicine, Vol. 62, no. 3, pp. 165 175.

[7] Huan Wang, Wynne Hsu, Kheng Guan Goh and Mong Li Lee. 2000. An effective approach to detect lesions in retinal images. Proc. IEEE Conf. Comput. Vis. Pattern Recogn., Hilton Head Island, SC, Vol. 2, pp. 181-187.

[8] Huiqi Li, Opas Chutatape, 2004. Automated Feature Extraction in Color Retinal Images by a Model Based Approach, IEEE Transactions On Biomedical Engineering, Vol. 51, no. 2, pp. 246-254.

[9] Lili Xu, Shuqian Luo, 2010. A novel method for blood vessel detection from retinal images, BioMedical Engineering OnLine, 9:14.

[10] Jagdish Nayak, P.Subbanna Bhat, Rajendra Acharya et al., 2008. Automated Identification of Diabetic Retinopathy Images using Digital Fundus Images. Journal of Medical Systems.32: 107-115.

[11] Neera Singh and Ramesh chandra tripathi, 2010. Automated Early Detection Of Diabetic Retinopathy Using Image Analysis Techniques. International Journal of Computer Applications. Vol.8,No.2, pp. 0975-8887.

[12] M. Niemeijer, B. V. Ginneken, S. R. Russell, M. Suttorp, and M. D. Abramoff, 2007. "Automated detection and differentiation of drusen, exudates and cotton-wool spots in digital color fundus photographs for diabetic retinopathy diagnosis," Invest. Ophthalmol. Vis. Sci., vol. 48, pp. 2260-2267. 
[13] Rema M. and Pradeepa R., 2007. Diabetic retinopathy: an Indian perspective. Indian Journal of Medical Research, Vol. 125, no. 3, pp. 297.

[14] Saiprasad ravikumar et al., 2007. Automated Feature Extraction For Early Detection Of Diabetic Retinopathy In Funds Image. IEEE international conference on Image Processing, pp 139-142.

[15] C. I. Sánchez, R. Hornero, M. I. López, J. Poza, 2004, Retinal Image Analysis to Detect and Quantify Lesions Associated with Diabetic Retinopathy, Proceedings of the 26th Annual International Conference of the IEEE EMBS, September 1-5.

[16] Sinthanayothin C, Boyce JF, Williamson TH, Cook HL, Mensah E, Lal S, Usher D., 2002. Automated detection of diabetic retinopathy on digital fundus images. Diabetic Medicine.19(2), 105-12.

[17] Usher D, Dumskyj M, Himaga M, Williamson TH, Nussey S, Boyce J, 2004. Automated detection of diabetic retinopathy in digital retinal images: a tool for diabetic retinopathy screening. Diabet Med 21, 84-90.

[18] Prof B. Venkatalakshmi, V.Saravanan. GJenny Niveditha 2011, Graphical User Interface for Enhanced Retinal Image Analysis for Diagnosing Diabetic Retinopathy, $3^{\text {rd }}$ International Conference on Communication Software and Networks (ICCSN), 610-613.

[19] Thomas Walter, Jean-Claude Klein, Pascale Massin, and Ali Erginay, 2002. A contribution of image processing to the diagnosis of diabetic retinopathy-detection of exudates in color fundus images of the human retina. Medical Imaging, IEEE Transactions on, Vol. 21, no. 10, pp. 1236-1243.

[20] V.Vijaya Kumari, N.SuriyaNarayanan, 2010, Diabetic Retinopathy-Early Detection Using Image Processing Techniques, International Journal on Computer Science and Engineering, Vol. 02, No. 02, 357-361. 